\title{
Analise comparativa do regime hídrico e térmico de dois sítios experimentais no Pampa
}

Comparative analysis of water and thermal regime of two experimental sites in Pampa.

\section{Claudio Alberto Teichrieb ${ }^{1}$, Pablo Eli Soares de Oliveira ${ }^{2}$, Tamires.Zimmer ${ }^{3}$, Cristiano maboni ${ }^{4}$, Daniel Michelon dos Santos ${ }^{5}$, Débora Regina Roberti ${ }^{6}$, Hans Rogério Zimermann ${ }^{7}$}

\author{
${ }^{1}$ Doutor em Física, Departamento de Física, UFSM, Santa Maria, Brasil \\ teichrieb@gmail.com \\ ${ }^{2}$ Doutorando em Meteorologia, Departamento de Meteorologia, UFSM, Santa Maria, Brasil \\ ${ }^{3}$ Mestranda em Física, Departamento de Física, UFSM, Santa Maria, Brasil \\ ${ }^{4}$ Mestrando em Física, Departamento de Física, UFSM, Santa Maria, Brasil \\ ${ }^{5}$ Doutor em Física, Departamento de Engenharia, UNIPAMPA, Alegrete, Brasil \\ ${ }^{6}$ Doutora em Física, Departamento de Física, UFSM, Santa Maria, Brasil \\ ${ }^{7}$ Doutor em Física, Departamento de Física, UFSM, Santa Maria, Brasil
}

\begin{abstract}
Resumo
Nos últimos 15-20 anos tem aumentado muito as pesquisas sobre o problema das alterações climáticas, necessitando-se uma demanda por medições confiáveis, de absorção e emissão de dióxido de carbono, metano, bem como o impacto nas reservas hídricas. No bioma Pampa se encontram as maiores extensões de campo natural contínuo, necessitando um monitoramento do regime hídrico e térmico no solo. O conteúdo de água no solo apresenta variabilidade espacial e temporal, afetando diversos processos hidrológicos e a determinação deste faz-se necessária, uma vez que o solo armazena e fornece água e nutrientes para as plantas, envolvendo desta forma as relações água-solo-planta-atmosfera. Neste trabalho, comparou-se o comportamento do conteúdo de água no solo nas profundidades de 10, 30 e $50 \mathrm{~cm}$, da temperatura no solo nas profundidades de 5,15 e $30 \mathrm{~cm}$, do fluxo de calor no solo instalado a $10 \mathrm{~cm}$ de profundidade e determinou-se a condutividade térmica, nos dois sítios experimentais no bioma Pampa, para o período de 01/01/2015 a 31/06/2015. Há diferenças entre os sítios, tanto na capacidade de reter umidade no solo e como na capacidade de armazenar energia no solo para o período analisado.
\end{abstract}

Palavras-chave: Umidade do solo, Temperatura do solo, Fluxo de calor do solo, Condutividade térmica, Pampa

\begin{abstract}
In the last 15-20 years has greatly increased research on the problem of climate change, necessitating a demand for reliable measurements of absorption and emission of carbon dioxide, methane, as well as the impact on water resources. In the biome Pampa are the largest continuous natural field extensions, requiring a monitoring of water and temperature regime on the ground. The water content of the soil has spatial and temporal variability affecting many hydrological processes and determining this is needed since the soil store and provide the water and nutrients for the plants, thus involving relationships water-soil-plant-atmosphere. In this work, we compared the water content behavior of the soil at depths of 10, 30 and $50 \mathrm{~cm}$, the temperature of the soil at depths of 5, 15 and $30 \mathrm{~cm}$, heat flux in soil installed $10 \mathrm{~cm}$ deep and the thermal conductivity was determined in two experimental sites in the Pampa biome, for the period 01.01.2015 to 06.31.2015. It was found that there are differences between the sites in the capacity to retain moisture in the soil and in the ability to store energy in the soil for the study period.
\end{abstract}

Keywords: Soil moisture, soil temperature, soil heat flow, thermal conductivity, Pampa. 


\section{Introdução}

O território brasileiro está dividido em seis diferentes biomas terrestres: Amazônia, Cerrado, Mata Atlântica, Caatinga, Pampa e Pantanal. O bioma Pampa está restrito ao estado do Rio Grande do Sul, onde ocupa uma área de 176.496 $\mathrm{km}^{2}$ (IBGE, 2004), isto corresponde a $63 \%$ do território estadual e a $2,07 \%$ do território brasileiro. O Pampa possui uma flora composta, majoritariamente, pela vegetação de pastagens naturais, entremeadas de matas de galeria (OVERBECK et al., 2007). É no bioma Pampa que se encontram as maiores extensões de campo natural contínuo. Estes campos constituem a matriz da vegetação do bioma, apresentando-se entremeados por florestas ao longo dos cursos dos rios (BOLDRINI et al., 2010).

$\mathrm{O}$ aumento das pesquisas sobre o problema das alterações climáticas nos últimos 15-20 anos tem aumentado a demanda por medições confiáveis, de absorção e emissão de dióxido de carbono, metano, bem como o impacto nas reservas hídricas. O conhecimento dos regimes hídrico e térmico de um local fornece informações importantes sobre as relações soloplanta-atmoefera.

O conteúdo de água no solo apresenta variabilidade espacial e temporal, afetando diversos processos hidrológicos. A determinação do conteúdo de água do solo faz-se necessária, uma vez que o solo armazena e fornece água e nutrientes para as plantas, envolvendo desta forma as relações água-solo-planta-atmosfera.

Medidas de temperatura no solo e na sua superfície são importantes, pois fornecem informações para conhecermos a transferência de energia na forma de calor em diferentes coberturas vegetais do solo. As variações da temperatura do solo nas diferentes camadas têm influencia na evapotranspiração e nos processos de troca de energia. O fluxo de calor no solo ocorre pela transferência de calor por condução, sendo a condutividade térmica a propriedade essencial que controlará o fluxo de calor no solo.

O objetivo deste trabalho é comparar o regime hídrico (umidade do solo) e térmico de dois sítios experimentais localizados no bioma Pampa, no período de 01/01/2015 a 30/06/2015.

\section{Materiais e métodos}

Os dados utilizados neste trabalho foram obtidos a partir de medias realizadas em duas estações micrometeorológicas da rede SULFLUX (http://www.sulflux.ufsm.br), sendo uma localizada no município de Pedras Altas - RS (PA) e outra no município de Santa Maria - RS (SM). A Figura 1 mostra a localização dos dois sítios experimentais inseridos no bioma Pampa.

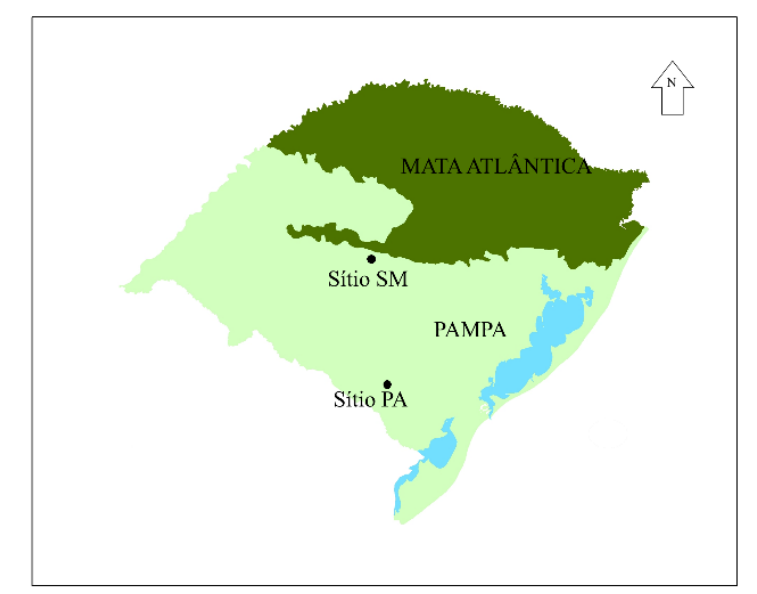

Figura 1 - Mapa indicando a localização dos sítios experimentais Santa Maria (SM) e Pedras Altas (PA). Fonte: Adaptação de Mapas de Biomas do Brasil IBGE,2004

A Tabela 1 mostra algumas informações comparativas dos dois sítios experimentais analisados neste estudo.

Foram utilizados dados do período de 01/01/2015 a 30/06/2015 e as medidas foram obtidas por sensores de mesmo modelo para cada uma das variáveis em ambos os sítios.

$\mathrm{Na}$ literatura encontra-se que o solo pode ser definido em três fases, a sólida que é constituída pelas partículas que compõem a matriz do solo, a líquida que é constituída pela água e a gasosa constituída pelo ar e pelo vapor de água. (BUSKE,2013). Segundo Bernardo et al. (2006) o volume da parte sólida pode ser considerada fixa, enquanto os gases e a solução (água e nutrientes) dividem o espaço poroso do solo. Conforme Kaiser (2010) o solo é considerado o armazenados de água para as plantas e ajuda a regular o ciclo hidrológico no ambiente. 
Tabela 1: Detalhes dos Sítios PA e SM

\begin{tabular}{lll}
\hline Sítio & PA & SM \\
\hline Localidade & Pedras & Santa \\
& Altas/RS & Maria/RS \\
Longitude $\left(^{\circ}\right)$ & $-53,5339$ & $-53,7597$ \\
Latitude $\left(^{\circ}\right)$ & $-31,7258$ & $-29,7241$ \\
Altitude $(\mathrm{m})$ & 375 & 88 \\
Tipo de solo & Argissolo/ & Argissolo/ \\
& Vermelho/ & Vermelho \\
& Amarelo & Distrófico \\
& Distrófico & \\
$\begin{array}{l}\text { Textura do solo } \\
\text { Precipitação } \\
\text { annual (mm) }\end{array}$ & Média/argilosa & Argilosa \\
Temperatura do & 1350 & 1617 \\
ar (min,max) $\left[\left[^{\circ}\right]\right.$ & & \\
\hline
\end{tabular}

A calibração dos dados para o conteúdo de água volumétrica (VWC) no solo foi realizada utilizando-se a equação quadrática fornecida pelo fabricante das sondas TDR CS 616. Utilizouse a seguinte equação: $V W C=-0,0663-0,0063 * P$ $+0,0007 * \mathrm{P}^{2}$, onde $\mathrm{P}$ é dado em microssegundos e é a leitura registrada no datalogger. Os sensores eram novos e não foi realizada intercomparação entre os sensores.

O conteúdo de água no solo foi medido por sondas TDR, modelo CS616 - Campbell Scientific, Inc. As sondas medem $30 \mathrm{~cm}$ de comprimento e foram posicionadas horizontalmente no solo, nas profundidades de 10,30 e $50 \mathrm{~cm}$. O TDR é um sensor que fornece o conteúdo de água no solo, através da medida da constante dielétrica do solo, ou seja, o TDR estabelece a constante dielétrica do solo, através da medição do tempo para que um pulso eletromagnético emitido entre duas barras condutoras paralelas de um determinado comprimento, cravadas no solo, alcance o seu final e voltar ao ponto de emissão. Desta forma, a informação de teor de água é derivada da sensibilidade da sonda com a constante dieléctrica do meio que circunda as hastes de sonda (Manual Campbell- CS616).

A temperatura do solo foi medida com termômetros que usam um termistor (semicondutor sensível á temperatura) para realizar as medidas das temperaturas, sendo utilizado o sensor modelo 108 - Campbell Scientific, Inc. Os termômetros foram posicionados horizontalmente no solo, nas profundidades de 5,15 e $30 \mathrm{~cm}$.

O fluxo de calor no solo mediu-se com o sensor modelo HFP01- Hukseflux, que gera um sinal de voltagem que é proporcional ao fluxo de calor do meio circundante neste caso no solo. Foi instalado a $10 \mathrm{~cm}$ de profundidade.

A temperatura da superfície do solo foi medida com o sensor SI-111- Campbell Scientific, Inc., que é um sensor de temperatura infravermelho que fornece a temperatura da superfície de um objeto (solo) sem estar em contato com ele. Foi instalado na base da torre a cerca de $30 \mathrm{~cm}$ de altura do solo, posicionado para que tenha um campo de visão de um semi ângulo de $22^{\circ}$, conforme manual de instruções do fabricante.

Os sensores estão instalados nas mesmas profundidades/alturas nos dois sítios.

Os sensores foram conectados a um sistema automático de aquisição de dados CR1000 (datalogger), programado para fazer medidas a cada 3 segundos e armazenar a média a cada 2 minutos para SM e média a cada 1 minuto para PA. Para comparar os dados dos dois sítios, foram realizadas médias de dois minutos nos dados de PA, ficando assim com a mesma frequência de SM. Também foram calculadas médias diárias.

Outro fator analisado foi á condutividade térmica do solo, que é a capacidade do solo em transmitir energia, ou seja, representa a velocidade na qual a energia passa através de uma camada do solo quando existe um gradiente de $1{ }^{\circ} \mathrm{C} / \mathrm{cm}$

Determinou-se a condutividade térmica para o solo nos dois sítios utilizando a equação do fluxo vertical de calor no solo (equação de Fourier):

$$
G=-K s \frac{\partial T}{\partial z}
$$

onde $\mathrm{G}$ é o fluxo de calor no solo $\left(\mathrm{W} \mathrm{m}^{-2}\right)$, Ks é a condutividade térmica do solo $\left(\mathrm{W} \mathrm{m}^{-1} \mathrm{~K}^{-1}\right)$ e

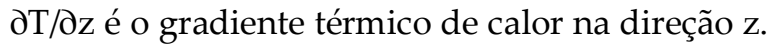
Sendo G e $\partial \mathrm{T} / \partial z$ conhecidos, podemos determinar a condutividade térmica reescrevendo a equação (1):

$$
K s=-G \frac{\left(z_{2}-z_{1}\right)}{\left(T_{2}-T_{1}\right)}
$$


onde $T_{2}$ e $T_{1}$ são as temperaturas do solo nos níveis de profundidades $\mathrm{Z}_{2}$ e $\mathrm{z}_{1}$ respectivamente, sendo $\mathrm{Z}_{2}>\mathrm{z}_{1}$. Determinou-se a condutividade térmica utilizando os termômetros de solo instalados a 5 e $10 \mathrm{~cm}$ de profundidade, e o fluxo foi medido a $10 \mathrm{~cm}$

\section{Resultados e discussões}

As Figuras 2 e 3 fornecem a resposta média diária do conteúdo volumétrico de água no solo, em três níveis de profundidades (10, 30 e $50 \mathrm{~cm}$ ) ao total diário precipitado para os sítios de Santa Maria (SM) e Pedras Altas (PA) respectivamente.

Para os 181 dias analisados (dia Juliano dj1 até o dia Juliano dj181 de 2015), observou-se que em SM a umidade do solo nos $50 \mathrm{~cm}$ de profundidade apresenta uma variação de umidade de 29 a $37 \%$, nos $30 \mathrm{~cm}$ varia de 22,5 a $33,5 \%$ e a $10 \mathrm{~cm}$ tem variação de 20 a $34 \%$ e no sítio PA, nos $50 \mathrm{~cm}$ a umidade varia de 7 a $29 \%$, nos $30 \mathrm{~cm}$ varia de 5,5 a $34 \%$ e em $10 \mathrm{~cm}$ varia de 6 a $32.5 \%$. Portando, para o sítio SM a diferença entre os valores extremos foi de 8,11 e $14 \%$ para as profundidades de 50,30 e $10 \mathrm{~cm}$ respectivamente e para o sítio PA essa diferença foi de 22, 28,5 e 26,5 para as profundidades 50, 30 e $10 \mathrm{~cm}$ respectivamente.

Neste período observa-se que a maior estiagem teve duração de 20 dias em SM, do dia Juliano dj64 ao dia dj84, e em PA a maior estiagem durou 22 dias do dj66 ao dj88. Podemos verificar que em SM, no nível de $50 \mathrm{~cm}$ a umidade sempre esteve superior a $30 \%$ e em apenas dois pequenos períodos a umidade do solo, para os níveis de 10 e $30 \mathrm{~cm}$, esteve abaixo dos $25 \%$ (dj40 a dj50 e dj73 a DJ 83). Já em PA, em apenas dois pequenos períodos a umidade do solo, para os três níveis, esteve acima dos 25 $\%$ (dj1 a dj17 e dj160 adj165).

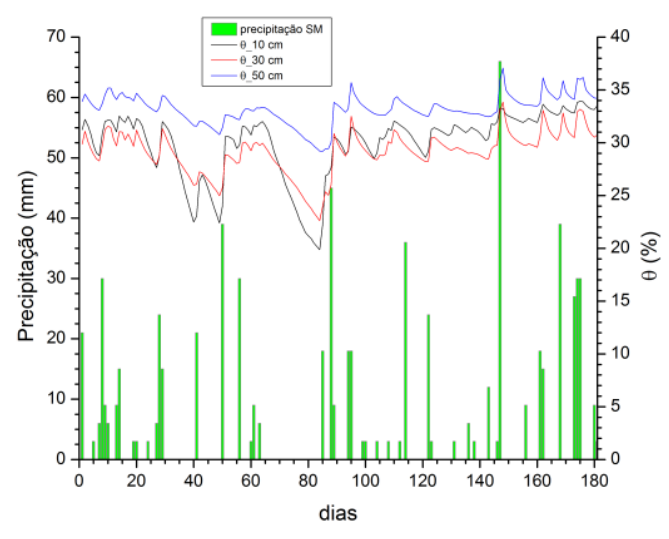

Figura 2 - Precipitação e conteúdo volumétrico de água do solo $(\theta)$ em três níveis de profundidade, 10, 30 e $50 \mathrm{~cm}$ para o sítio experimental SM (Santa Maria/RS). Média diária.

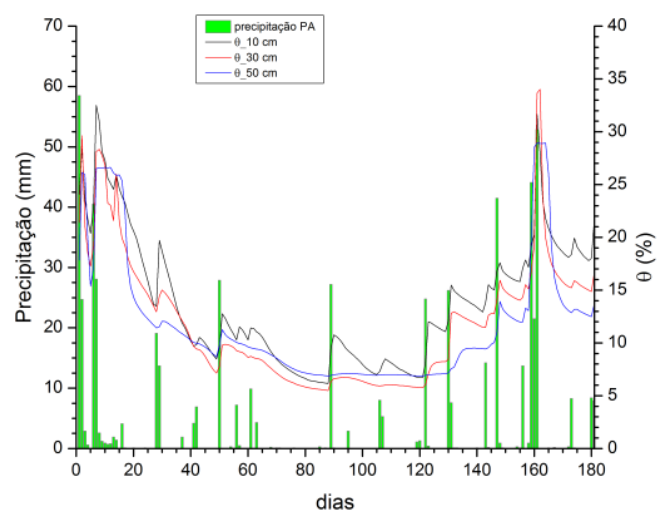

Figura 3 - Precipitação e conteúdo volumétrico de água do solo $(\theta)$ em três níveis de profundidade, 10, 30 e $50 \mathrm{~cm}$ para o sítio experimental PA (Pedras Alta/RS). Média diária.

A Figura 4 destaca a comparação da dinâmica do conteúdo volumétrico de água do solo para cada um dos três níveis de profundidade (10, 30 e $50 \mathrm{~cm}$ ) para os sítios $\mathrm{SM}$ e PA. Nesta comparação fica mais visível a diferença na capacidade de retenção da agua pelo solo em cada sítio.

No nível de $50 \mathrm{~cm}$ no sítio SM, a umidade do solo se mantém acima dos $30 \%$ e em PA, com exceção dos primeiros 20 dias e dos 20 dias finais, a umidade ficou abaixo dos $15 \%$, destacando o período do dia dj80 ao dj130, onde a umidade ficou constante em $7 \%$. No nível de $30 \mathrm{~cm}$ no sítio SM, a umidade esteve acima dos $35 \%$ na grande maioria dos dias analisados, e para PA assim como no nível de $50 \mathrm{~cm}$, a umidade ficou a maior parte do período abaixo 
dos $15 \%$. No nível de $10 \mathrm{~cm}$ no sítio $\mathrm{SM}$, a umidade ficou acima dos $25 \%$ e próximo de 30 $\%$, exceto para dois períodos de estiagem, onde a umidade baixou para próximo dos $20 \%$. Em PA, nos $10 \mathrm{~cm}$, a umidade ficou a metade do período acima dos $15 \%$ e outra metade abaixo dos $15 \%$.

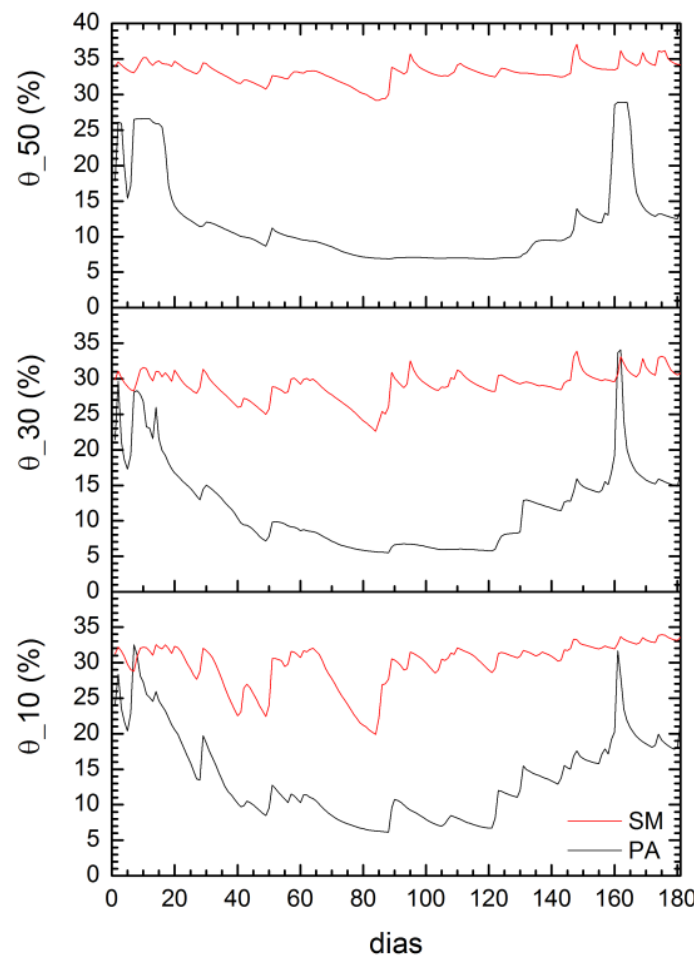

Figura 4 - Umidade do solo $(\theta)$ em 3 níveis de profundidade, 50, 30 e $10 \mathrm{~cm}$ para os sítios PA (Pedras Alta/RS) linha preta e SM (Santa Maria/RS) linha vermelha. Média diária.

Portanto, nessa analise direta, podemos ver claramente que o sítio experimental SM apresenta uma maior capacidade de reter a água no solo, mantendo uma variação na umidade menor em relação a PA.

Os fatores que influenciam na variabilidade do conteúdo de água no solo são: a topografia, as propriedades do solo, tipo e densidade da vegetação, lençol freático precipitação e radiação solar e outras variáveis meteorológicas (MEDEIROS; CLARKE, 2007; CANTÓN et al., 2004). De acordo com Santos (2010), há uma dificuldade em se identificara a importância relativa da cada um desses fatores, devido as suas múltiplas e mútuas influencias na umidade do solo e assim, sugere que as relações entre os fatores descritos anteriormente e a umidade do solo sejam analisadas em varias escalas e locais diferentes.

Para os sítios de SM e PA os fatores que levam a acreditar que mais influenciaram nos resultados foram: as diferenças no relevo dos dois sítios, as propriedades físicas do solo e a vegetação. O sitio PA está em ema localização topográfica mais alta e o sítio SM esta localizada próximo a uma várzea. A textura do solo difere nos dois sítios, sendo argiloso no sitio $\mathrm{SM}$ e média em PA, o que influencia na penetração e retenção de água no solo. A vegetação de SM é mais densa, mantendo o solo mais protegido. Outro fator observado mostra que a precipitação foi mais bem distribuída no sítio de SM e a infiltração da água ocorre mais rápido nas camadas inferiores, mantendo desta forma o solo mais úmido.

Na Figura 5 esta representada a média diária da temperatura do solo em três níveis de profundidade (30, 15 e $5 \mathrm{~cm}$ ) e a temperatura da superfície do solo, de cima para baixo respectivamente.

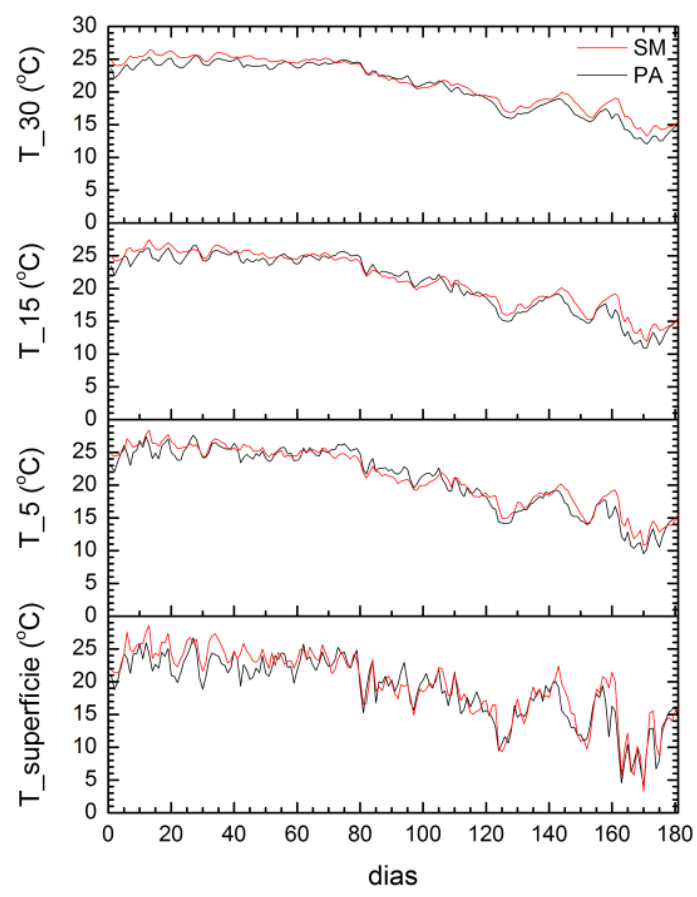

Figura 5 - Temperatura do solo (T) para três níveis de profundidade, 30, 15 e $5 \mathrm{~cm}$ e temperatura da superfície do solo para os sítios experimentais de PA (Pedras Alta/RS) linha preta e SM (Santa Maria/RS) linha vermelha. Média diária.

O sítio de SM apresenta a média diária da temperatura do solo maior para os três níveis na 
maior parte do período analisado, exceto entre os dias dj72 e dj 106, onde o sítio PA apresentou valores maiores de temperatura. As maiores temperaturas são verificadas no período do verão e foi decrescendo lentamente até os dias próximos do inverno. Como é de se esperar, as temperaturas do solo são maiores no período diurno e quanto mais próximo o sensor estiver na superfície. A amplitude da temperatura do sítio SM é menor que o de PA em todos os níveis.

A Figura 6 mostra o fluxo de calor no solo para o período do estudo. Entende-se por fluxo de calor no solo como sendo uma fração do saldo de radiação que é transferida aos níveis inferiores do solo, dependendo da temperatura do solo em diferentes níveis de profundidade e da condutividade térmica do solo e geralmente, resulta em acréscimo de energia para o meio à noite, ou em diminuição, no período diurno (GALVANI et al.,2001).

O fluxo de calor no solo foi mais positivo no período mais quente e mais negativo no período mais frio, como é esperado. O sítio de PA teve a maior amplitude do fluxo positivo de calor no solo e o sítio SM teve o maior fluxo negativo.

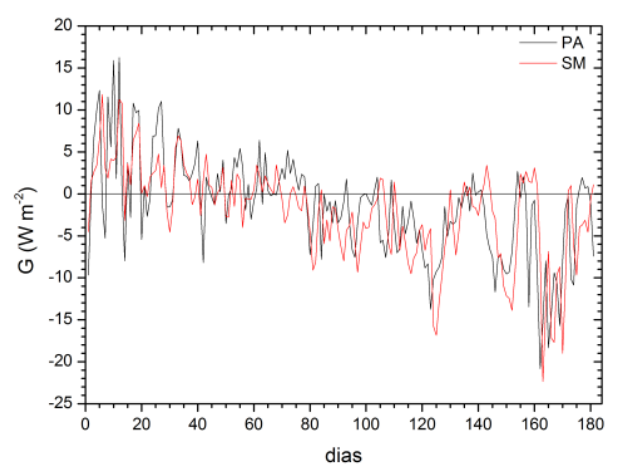

Figura 6 - Fluxo de calor no solo $(G)$ na profundidade de $10 \mathrm{~cm}$ para os sítios experimentais de PA (Pedras Alta/RS) linha preta e SM (Santa Maria/RS) linha vermelha. Média diária.

Determinou-se a condutividade térmica usando a equação (2) para os sítios de SM e PA, utilizando dados com uma frequência de 2 minutos. Para o sítio experimental de SM, encontrou-se $\mathrm{K}_{\mathrm{ssm}}=1,12 \mathrm{~W} \mathrm{~m}^{-1} \mathrm{~K}^{-1}$ e para PA obteve-se $\mathrm{K}_{\mathrm{spa}}=0,85 \mathrm{~W} \mathrm{~m}^{-1} \mathrm{~K}^{-1}$. Na literatura, para solo argiloso ( $40 \%$ de espaço poroso) seco e saturado tem-se 0,25 e $1,58 \quad W \quad m^{-1} \quad K^{-1}$ respectivamente (OKE, 1987). Sendo a condutividade térmica um processo de transferência de energia no qual a energia é transferida de molécula para molécula, sem que haja deslocamento das moléculas de sua posição original, por esta razão, normalmente os corpos mais densos apresentam mais facilidade para conduzir a energia, ou seja, a condutividade térmica do solo depende da textura, da porosidade e da umidade. Portanto, quando o solo é umedecido ocorre uma melhora na sua capacidade de conduzir energia pois, substitui-se o ar (quase um isolante) pela água, que conduz melhor a energia.

$\mathrm{O}$ resultado indica que o sítio de $\mathrm{SM}$, que tem a maior condutividade térmica, apresenta menor variação de temperatura na superfície e desta forma maior será o armazenamento do calor.

\section{Conclusões}

Podemos concluir que há uma diferença significativa no comportamento da umidade do solo entre os sítios experimentais de SM e PA. O sítio SM apresenta um solo que retém mais a umidade nos três níveis analisados, quando comparados ao sítio PA.

A temperatura média do solo no sítio SM foi maior em quase todo o período analisado e nas três profundidades, exceto para 32 dias consecutivos do dia Juliano 72 a 106, onde a temperatura do solo do sítio de PA foi maior. A amplitude da temperatura foi maior no sítio PA.

A condutividade térmica encontrada para $\mathrm{O}$ sítio de $\mathrm{SM}\left(\mathrm{Ks}=1,12 \mathrm{~W} \mathrm{~m}^{-1} \mathrm{~K}^{-1}\right)$ foi maior do que o de PA $\left(\mathrm{Ks}=0,85 \mathrm{~W} \mathrm{~m}^{-1} \mathrm{~K}^{-1}\right)$, indicando que o sitio de SM tem melhor capacidade de armazenar calor no solo.

\section{Agradecimentos}

Os autores agradecem a Fapergs e a CAPES pelo apoio financeiro.

\section{Referências}

Bernardo, S.; Soares, A. A.; Mantovani, E. C. (2006) Manual de irrigação. 8.ed - Viçosa: Ed. UFV. 625p.

Boldrini, I. I.; Ferreira, P. M. A.; Andrade, B. O.; Schneider, A. A.; Setubal, R. B.; Trevisan, R; 
Freitas, E.M. (2010). Bioma Pampa: diversidade florística e fisionômica. Porto Alegre, editora Pallotti. 64 p.

Buske, T. C. Comportamento da umidade do solo determinada por métodos expeditos. Dissertação de mestrado- Universidade federal de Santa Maria, Santa Maria, RS, Brasil, 66p, 2013.

CANTÓN, Y.; SOLÉ-BENET, A.; DOMINGO, F. (2004). Temporal and spatial patterns of soil moisture in semiarid badlands of SE Spain. Journal of Hydrology, n. 285, p. 199-214.

IBGE. (2004). Instituto Brasileiro de Geografia e Estatística. Acessado em 20/08/2015. Disponível em:

http://saladeimprensa.ibge.gov.br/noticias?view= noticia\&id $=1 \&$ busca $=1$ \&idnoticia $=169$

Galvani, E.; Escobedo, J. F.; Pereira, A. B. 2001. Balanço de radiação e fluxo de calor no solo em ambiente natural e protegido cultivado com pepineiro. Bragantia, Campinas, 60(2), 139-147.

Kaiser, D. R. (2010). Universidade federal de Santa Maria. Centro de Ciencias Rurais. Fundamentos da ciência do solo. Aulas práticas. Santa Maria. 112p.

MEDEIROS, J. D.; CLARKE, J. A. G. (2007) Variabilidade espacial do conteúdo de água no solo numa pequena bacia rural: Análise geoestatística. Revista Brasileira de Recursos Hídricos, v. 12, n. 1, p. 43-52.

Oke, T. R. (1987) Boundary Layer Climate. 2nd Edition, Routledge, $43-44$.
Overbeck, G. E. et al. (2007). Brazil's neglected biome: the South Brazilian Campos.

Perspectives in Plant Ecology, Evolution and Systematics, v.9, p.101-116.

SANTOS, T. E. M. (2010) Dinâmica espacial e temporal da umidade do solo em bacia experimental do semiárido pernambucano. 2010. 68 p. Tese (Doutorado em Agronomia, Ciência do Solo) - Universidade Federal Rural de Pernambuco, Recife. 


\title{
Analise comparativa do regime hídrico e térmico de dois sítios experimentais no Pampa
}

Comparative analysis of water and thermal regime of two experimental sites in Pampa.

\section{Claudio Alberto Teichrieb ${ }^{1}$, Pablo Eli Soares de Oliveira ${ }^{2}$, Tamires.Zimmer ${ }^{3}$, Cristiano maboni ${ }^{4}$, Daniel Michelon dos Santos ${ }^{5}$, Débora Regina Roberti ${ }^{6}$, Hans Rogério Zimermann $^{7}$}

\author{
${ }^{1}$ Doutor em Física, Departamento de Física, UFSM, Santa Maria, Brasil \\ teichrieb@gmail.com \\ ${ }^{2}$ Doutorando em Meteorologia, Departamento de Meteorologia, UFSM, Santa Maria, Brasil \\ ${ }^{3}$ Mestranda em Física, Departamento de Física, UFSM, Santa Maria, Brasil \\ ${ }^{4}$ Mestrando em Física, Departamento de Física, UFSM, Santa Maria, Brasil \\ ${ }^{5}$ Doutor em Física, Departamento de Engenharia, UNIPAMPA, Alegrete, Brasil \\ ${ }^{6}$ Doutora em Física, Departamento de Física, UFSM, Santa Maria, Brasil \\ ${ }^{7}$ Doutor em Física, Departamento de Física, UFSM, Santa Maria, Brasil
}

\begin{abstract}
Resumo
Nos últimos 15-20 anos tem aumentado muito as pesquisas sobre o problema das alterações climáticas, necessitando-se uma demanda por medições confiáveis, de absorção e emissão de dióxido de carbono, metano, bem como o impacto nas reservas hídricas. No bioma Pampa se encontram as maiores extensões de campo natural contínuo, necessitando um monitoramento do regime hídrico e térmico no solo. O conteúdo de água no solo apresenta variabilidade espacial e temporal, afetando diversos processos hidrológicos e a determinação deste faz-se necessária, uma vez que o solo armazena e fornece água e nutrientes para as plantas, envolvendo desta forma as relações água-solo-planta-atmosfera. Neste trabalho, comparou-se o comportamento do conteúdo de água no solo nas profundidades de 10,30 e $50 \mathrm{~cm}$, da temperatura no solo nas profundidades de 5,15 e $30 \mathrm{~cm}$, do fluxo de calor no solo instalado a $10 \mathrm{~cm}$ de profundidade e determinou-se a condutividade térmica, nos dois sítios experimentais no bioma Pampa, para o período de 01/01/2015 a 31/06/2015. Há diferenças entre os sítios, tanto na capacidade de reter umidade no solo e como na capacidade de armazenar energia no solo para o período analisado.
\end{abstract}

Palavras-chave: Umidade do solo, Temperatura do solo, Fluxo de calor do solo, Condutividade térmica, Pampa

\begin{abstract}
In the last 15-20 years has greatly increased research on the problem of climate change, necessitating a demand for reliable measurements of absorption and emission of carbon dioxide, methane, as well as the impact on water resources. In the biome Pampa are the largest continuous natural field extensions, requiring a monitoring of water and temperature regime on the ground. The water content of the soil has spatial and temporal variability affecting many hydrological processes and determining this is needed since the soil store and provide the water and nutrients for the plants, thus involving relationships water-soil-plant-atmosphere. In this work, we compared the water content behavior of the soil at depths of 10, 30 and $50 \mathrm{~cm}$, the temperature of the soil at depths of 5,15 and $30 \mathrm{~cm}$, heat flux in soil installed $10 \mathrm{~cm}$ deep and the thermal conductivity was determined in two experimental sites in the Pampa biome, for the period 01.01.2015 to 06.31.2015. It was found that there are differences between the sites in the capacity to retain moisture in the soil and in the ability to store energy in the soil for the study period.
\end{abstract}

Keywords: Soil moisture, soil temperature, soil heat flow, thermal conductivity, Pampa. 